\title{
HISTORIA DEL FUEGO Y LA VEGETACIÓN EN UNA SECUENCIA HOLOCENA DEL PIRINEO CENTRAL: LA BASA DE LA MORA
}

\author{
L. LASHERAS-ÁLVAREZ*, A. PÉREZ-SANZ, \\ G. GIL-ROMERA, P. GONZÁLEZ-SAMPÉRIZ, \\ M. SEVILLA-CALLEJO, B. VALERO-GARCÉS
}

Dpto. de Procesos Geoambientales y Cambio Global, Instituto Pirenaico de Ecología, CSIC, Campus Aula Dei, Av. Montañana 1005, 50059 Zaragoza.

\begin{abstract}
RESUMEN. La importancia del estudio de la actividad del fuego en el pasado está en la necesidad de comprender los efectos que el Cambio Global puede tener sobre la misma, tanto en la actualidad como en el futuro. Estudiar cómo se relaciona el fuego con los ecosistemas y el clima en escalas temporales lo suficientemente largas permite conocer la respuesta de la vegetación a esta perturbación y definir los umbrales que provocan cambios irreversibles en la composición vegetal. Este trabajo estudia la dinámica del fuego durante el Holoceno en el entorno de la Basa de la Mora, lago de origen glaciar situado en el Macizo de Cotiella, Pirineo Central. La historia del fuego se ha reconstruido a partir de la identificación de partículas de carbón fósil a lo largo de la secuencia sedimentaria extraída del lago, que cubre los últimos 10000 años de historia. Se ha observado que la mayor actividad del fuego se produce entre 8.1 y 4 cal $\mathrm{Ka}$ BP, seguida de una fase de menor actividad entre 3.2 y 1.7 cal Ka BP. Estas fluctuaciones se deben principalmente a dos razones: la cantidad de biomasa disponible y susceptible de ser quemada, y la existencia de un clima favorable para la ocurrencia de incendios. En ese sentido, se han observado correlaciones positivas de la actividad del fuego, por un lado, con Betula y Corylus como taxones ligados a la mayor producción de biomasa, y por otro, con agrupaciones de taxones adaptados a la aridez como indicadores de climas favorables a la ocurrencia de incendios. Por último, a partir de 1.7 cal Ka BP se registra en la secuencia una mayor incidencia del fuego, probablemente vinculada a la intensificación de la acción antropogénica a nivel regional.
\end{abstract}

History of fire and vegetation in a Holocene sequence of the Central Pyrenees: the Basa de la Mora lake

ABSTRACT. The importance of studying past fire regimes is derived from the need to understand the effects of current Global Change on present and future 
fire activity. Studying the relationship between long-term fire ecology, ecosystems and climate allows us to understand how vegetation responds to this perturbation and to define the thresholds triggering irreversible changes in the vegetation. This work presents the last 10000 years of vegetation change and fire activity on a high altitude ecosystem from the Pyrenees range: the mountain lake Basa de la Mora located in the central southern Pyrenees. Holocene fire activity has been inferred through fossil carbon particle identification in the sedimentary sequence obtained in this lake. We observe that the strongest Holocene fire activity in the area occurred between 8.1 and 4 cal Kyr BP. This period was followed by a phase, between 3.2 and 1.7 cal Kyr BP, where almost no fire activity was recorded. These changes seem to be due to biomass availability and fire-conductive climate conditions. Positive correlation has been found between fire activity and Betula, Corylus, as the key fueling taxa and groups of ariditytolerant taxa. Additionally, fire dynamics seems coupled to higher anthropogenic activities after 1.7 cal Kyr BP.

Palabras clave: historia del fuego, ecología de la perturbación, microcarbón fósil, Holoceno, Pirineos.

Key words: fire history, disturbance ecology, fossil micro acharcoal, Holocene, Pyrenees.

Enviado el 6 de noviembre de 2012 Aceptado el 13 de enero de 2013

* Correspondencia: Departamento de Procesos Geoambientales y Cambio Global, Instituto Pirenaico de Ecología, CSIC, Campus Aula Dei, Av. Montañana 1005, 50059 Zaragoza. E-mail: laulasheras@ipe.csic.es

\section{Introducción}

El fuego ha sido denominado como un "herbívoro global" por Bond et al. (2005) aludiendo al importante papel que tiene en la formación de los ecosistemas y paisajes que conocemos hoy en día (Pausas y Keeley, 2009). El fuego puede ser considerado como uno de los agentes perturbadores que mayores efectos provocan sobre los ecosistemas, habiendo influido sobre los ciclos biogeoquímicos desde el origen de la vegetación terrestre y siendo reconocido como uno de los principales componentes del cambio global (Clark, 1988; Thonicke et al., 2001; Vannière et al., 2008; Bowman et al., 2009). Por ejemplo, el fuego es responsable de los cambios adaptativos en la vegetación de las plantas $\mathrm{C}_{4}$ (Bond y Keeley, 2005), debido al aumento de la frecuencia de incendios durante el periodo Terciario (Keeley y Rundel, 2005). Además, el fuego puede determinar tanto el estado de sucesión de la vegetación y su edad, como su distribución y composición (Niklasson y Granstrom, 2000; Thonicke et al., 2001). Por ello, la reconstrucción temporal y espacial de la actividad del fuego tiene una gran importancia a la hora de interpretar el paisaje.

Para la producción y propagación de un fuego es necesario que exista suficiente biomasa combustible, además de unas determinadas condiciones de temperatura y 
humedad (Patterson et al., 1987; Carcaillet et al., 2001; Thonicke et al., 2001). Por ello, debe existir una cierta estacionalidad en el clima, donde se den periodos secos en los que el combustible potencial, la biomasa, se convierta en combustible disponible (Pausas y Keeley, 2009). El clima Mediterráneo presenta, en este sentido, unas características de estacionalidad idóneas para la ocurrencia de incendios. Por otra parte, los cambios en el clima conllevan alteraciones en el régimen de incendios a través del control directo de la humedad y la temperatura, y de forma indirecta, de la composición de la vegetación, alterando la inflamabilidad de la madera. De esta forma, el clima puede contribuir directamente al aumento del riesgo de incendios, como ocurre durante años cálidos y secos (Pyne et al., 1984; Piñol et al., 1998).

Por todas estas razones, ante el escenario de aumento de la temperatura global en el que nos encontramos (Mann et al., 1999; IPCC, 2007), donde la gestión de los incendios puede ser de gran importancia a la hora de mantener la biodiversidad y el paisaje en su conjunto (Conedera et al., 2009; Fisher et al., 2009; Driscoll et al., 2010), resulta esencial el estudio del régimen de incendios en el pasado y sus relaciones con la vegetación y el clima. Entender las relaciones en escalas temporales largas nos ayudará a comprender qué papel desempeña el fuego en los ecosistemas, qué respuesta da la vegetación, y qué posibles efectos puede tener sobre el régimen de incendios un cambio en las condiciones climáticas (Whitlock et al., 2010). El estudio de los paleoincendios es aún más relevante en las zonas de alta montaña, en donde los ecosistemas no son particularmente resilientes a las perturbaciones profundas (Gotfried et al., 2012) y las fluctuaciones en la actividad del fuego pueden hacer peligrar tanto su diversidad florística como paisajística.

Por ello, el objetivo principal de este estudio es entender las relaciones existentes entre vegetación, clima y dinámica del fuego durante el Holoceno en un ecosistema de montaña. Este objetivo general se despliega a su vez en dos objetivos particulares:

- Reconstruir la historia regional del fuego, definiendo los periodos de máxima y mínima actividad, a la luz de los cambios climáticos ocurridos durante el Holoceno.

- Analizar la respuesta de la vegetación a los diferentes periodos de actividad del fuego, así como al potencial efecto que las fluctuaciones de biomasa hayan podido tener sobre la dinámica de los incendios.

Para el análisis de estas relaciones, este trabajo emplea técnicas de análisis de partículas de microcarbón fósiles, como parámetro indicador de la ocurrencia de fuegos en el pasado, así como la paleopalinología como herramienta para la reconstrucción de la vegetación. Las partículas de carbón resultan de la combustión incompleta de tejido vegetal que quedan acumuladas tras un incendio (Patterson et al., 1987), y mediante su cuantificación se puede estimar la importancia que tuvo el fuego a lo largo de una secuencia fósil. La comparación directa del espectro polínico y los microcarbones encontrados en la muestra de un sedimento nos permiten conocer la respuesta de la vegetación ante la intensificación del fuego, o los cambios en la ocurrencia de incendios 
debidos a variaciones de composición de la vegetación y al clima (Clarck, 1988; Sadori y Giardini, 2007).

En la vertiente norte del Pirineo central se han llevado a cabo estudios que reconstruyen los regímenes de incendios de manera local (Rius et al., 2009, 2011, 2012). Sin embargo, el único estudio existente hasta el momento de reconstrucción de dinámica de incendios regionales en el Pirineo meridional es el realizado por Bal et al. (2011), que describe la dinámica del fuego durante los últimos 3300 años, no habiendo hasta el momento datos que cubran todo el Holoceno, y que por tanto permitan comparar periodos en los que la intervención humana en la génesis de incendios no sea un actor a tener en cuenta. El trabajo aquí presentado, pretende precisamente esto, obtener datos que nos permitan conocer la dinámica del fuego en el Pirineo central a lo largo de los últimos 10000 años, de manera que se puedan comparar periodos con diferentes grados de acción antrópica y definir los moduladores climáticos de la actividad del fuego.

\section{Material y métodos}

\section{1. Área de estudio}

El ibón de Plan o Basa de la Mora (42³ 32’ N, 0 19’ E, 1914 m s.n.m.), se localiza en el Macizo de Cotiella, Pirineo central, dentro de las comarcas de Sobrarbe y Ribagorza (Huesca) (Fig. 1). Este macizo se encuentra delimitado al este por el barranco de la Aigüeta de Barabuens, por el río Cinca al oeste, al norte por el río Cinqueta y por el río Esera al sur, presentando un relieve abrupto con alturas que van desde los $2912 \mathrm{~m}$ s.n.m. -Pico Cotiella- hasta los 700 m s.n.m. en los fondos de valle. La Basa de la Mora se encuentra sobre la única cubeta glaciar presente en el macizo de Cotiella, que pudo llegar a los 300 metros de espesor de hielo durante el último máximo glaciar (Belmonte-Ribas, 1999). En la actualidad, la Basa de la Mora es un lago somero cuya lámina de agua no suele superar los 4 metros de profundidad, y posee una superficie de inundación de 6.3 hectáreas. Los aportes de agua al Ibón llegan a través de agua superficial por escorrentía y precipitación, mientras que la salida se realiza a través de un barranco (Barranco de L'Ibón) y por evaporación, funcionando como un sistema abierto. Predominan los materiales calizos en el sustrato, aflorando materiales yesíferos y ofíticos, que se erosionan fácilmente (Belmonte-Ribas, 1999).

La Basa de la Mora se encuentra en la transición de los pisos bioclimáticos alpino y subalpino, en el límite entre los ecosistemas típicamente Mediterráneos y Eurosiberianos que, junto con el abrupto relieve que rodea a este Ibón, conforman un paisaje caracterizado por importantes contrastes biogeográficos. El clima del área podría describirse como sub-mediterráneo con influencia continental. Las temperaturas medias máximas se registran durante los meses de julio y agosto alcanzando casi los $15^{\circ} \mathrm{C}$, mientras que las temperaturas medias más bajas apenas superan $\operatorname{los} 0^{\circ} \mathrm{C}$ en los meses de invierno, poniendo de manifiesto las fuertes variaciones termométricas estacionales en el Ibón. La precipitación media anual es de 1482 mm, típica de un clima oceánico, pero se distribuye mayoritariamente en primavera y otoño, siendo también de importancia las 


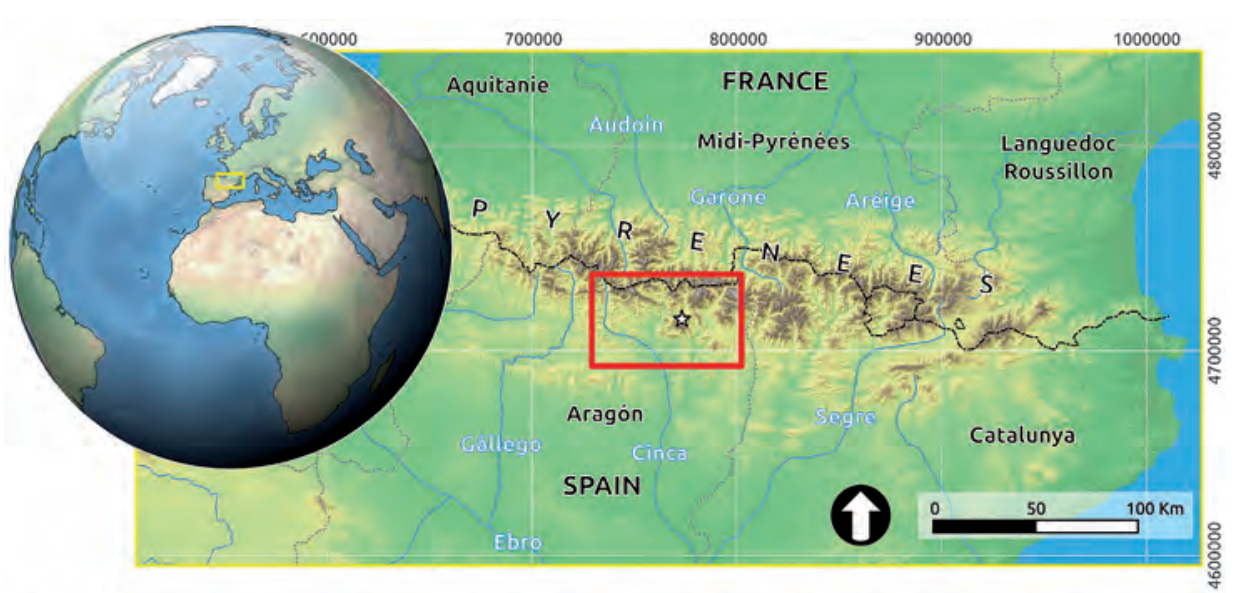

Legend
studied site
-.- international boundaries
regional boundaries
rivers
$=$ lakes \& reservoirs
urban areas
elevation (m.)
$=$ sea level
$=0$
$=500$
$=1000$
$=2000$
$=2500$
$=3000$
3500

Projection UTM 30 N Datum ETRS89 Topo. base source: Natural Earth \& National Topographic Map (BTN200)

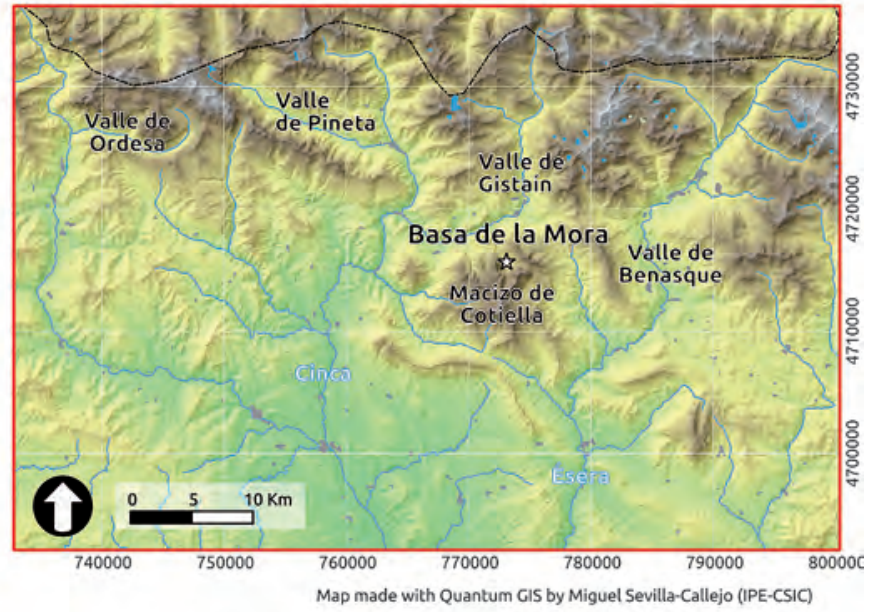

Figura 1. Mapa de localización de la Basa de la Mora.

tormentas convectivas de verano, características de todo el Pirineo Central, y que responden a un patrón más mediterráneo (Belmonte-Ribas, 2007).

Las vertientes de umbría del macizo de Cotiella presentan un bosque mixto bien desarrollado de pinares y abetales cuyas principales especies son Pinus sylvestris L., Pinus uncinata Mill., Abies alba Mill., Fagus sylvatica L.y Buxus sempervirens L. en el piso montano, mientras que en el piso subalpino encontramos Pinus uncinata y Rhododendron ferrugineum L. Por el contrario, las solanas se encuentran mayoritariamente deforestadas debido al pastoreo y la escasa capacidad de retención hídrica del terreno, por lo que las escasas formaciones arbóreas que podemos encontrar son de Quercus ilex L. subsp. Ballota (Desf.), Quercus faginea L., Pinus nigra Arn. y Pinus sylvestris. En los alrededores del ibón de la Basa de la Mora se localizan los pinares de pino negro 
(Pinus uncinata) mejor conservados en el macizo, además de Juniperus communis L. y Rhododendron sp. (Montserrat-Martí, 1989; Pérez-Sanz et al., 2011).

\subsection{Obtención de las muestras y cronología}

Los sondeos del sedimento lacustre del Ibón de la Basa de la Mora se recuperaron en una campaña de campo llevada a cabo por el equipo de Paleolimnología y Cambio Global del Instituto Pirenaico de Ecología-CSIC durante el verano de 2008. Mediante un estudio batimétrico se seleccionó el punto más profundo del lago tomándose dos sondeos paralelos, uno profundo y otro superficial, que se relacionaron mediante observación directa y siguiendo criterios geoquímicos. El sondeo profundo se obtuvo mediante un sondeador de pistón tipo UWITEC colocado sobre una plataforma flotante, y la parte superior de la secuencia se recuperó mediante un sondeador de gravedad. Finalmente la secuencia total correlacionada consta de 12.1 metros de sedimento.

Tras la datación de un total de 14 muestras, una de ellas mediante $\mathrm{Cs}^{137}$ y las 13 restantes mediante AMS $\mathrm{C}^{14}$, se determinó la edad absoluta del sondeo, que cubre los últimos 10000 años de historia (Pérez-Sanz et al., 2011, en prensa).

\section{Preparación de las muestras y obtención y análisis de los datos}

\section{POLEN}

La preparación de las muestras para el recuento de polen se ha realizado siguiendo el método de Moore et al. (1978) con algunas modificaciones propuestas por Dupré (1979). En este proceso se utilizan esporas de Lycopodium como marcador exótico, lo que permite calcular la concentración polínica y testar el proceso físico-químico del laboratorio. La identificación y recuento de los granos de polen existentes en cada muestra se realizó con ayuda de un microscopio óptico y consultando atlas de referencia y la palinoteca del IPE-CSIC. La representación de los porcentajes polínicos se hizo con ayuda del programa PSIMPOLL 4.27 (Bennett, 2009).

\section{MICROCARBONES}

La cantidad de partículas de carbón que se acumulan en un lago está relacionada con las características del fuego, y los procesos de transporte que ocasionan que las partículas acaben almacenándose en el sedimento lacustre (Whitlock y Larsen, 2001). Las partículas de un tamaño menor a $100 \mu \mathrm{m}$ son fácilmente elevadas y transportadas por el viento a largas distancias (Patterson et al., 1987; Clark, 1988), por ello se interpreta que estas partículas de microcarbón representan fuegos ocurridos en un área entre 20 y 100 $\mathrm{km}$ alrededor del lugar de estudio, representando así la ocurrencia de fuegos a nivel regional (Tiner y $\mathrm{Hu}, 2003$ ).

El recuento de microcarbones se realiza en las mismas preparaciones de polen, lo que implica que no resulte necesario realizar ningún otro protocolo de laboratorio, analizándose los dos parámetros de forma simultánea (Rhodes, 1998). Los microcarbones se 


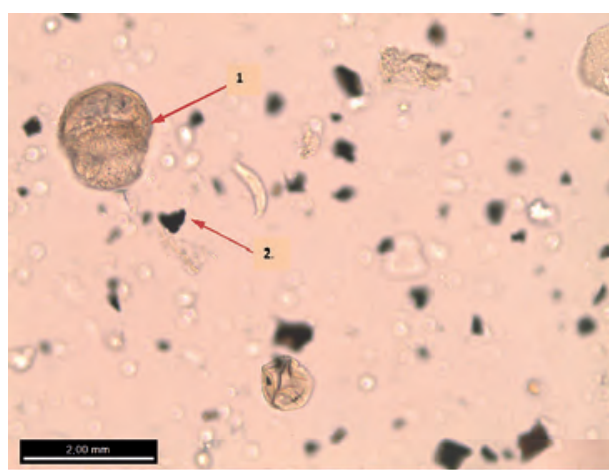

Figura 2. Fotografía (1) grano de polen de Pinus y (2) partículas de microcarbón.

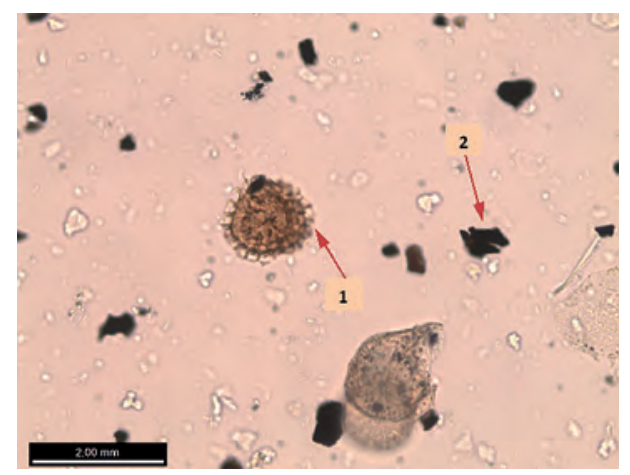

Figura 3. Fotografía (1) espora de Lycopodium y (2) partículas de microcarbón.

reconocen por ser de color negro, totalmente opacos y tener una forma angulosa (Fig. 2 y 3) (Rhodes, 1998). Así, siguiendo el método expuesto por Tinner y Hu (2003), sólo las partículas de microcarbón de un tamaño mayor a $10 \mu \mathrm{m}$ y menor a $150 \mu \mathrm{m}$ han sido tenidas en cuenta para el análisis. Las muestras han sido realizadas cada $5 \mathrm{~cm}$ a lo largo del sondeo, aumentando la resolución a $1 \mathrm{~cm}$ a partir de $1165.5 \mathrm{~cm}$, por ser la tasa de sedimentación baja a partir de este punto. Se han analizado un total de 142 muestras para polen y microcarbones.

Para poder explicar de manera robusta las relaciones existentes entre vegetación y dinámica de fuegos, resulta necesario llevar a cabo un estudio cuantitativo que, en primer lugar, implica transformar el número total de partículas de microcabón por peso de muestra $\left(\mathrm{n}^{\mathrm{o}}\right.$ partículas $\left./ \mathrm{g}\right)$, a área de carbón por peso de muestra $\left(\mathrm{mm}^{2} / \mathrm{g}\right)$, ya que los resultados obtenidos en mediciones de área son más coherentes que los basados únicamente en número de partículas (Patterson et al., 1987; Tinner y Hu, 2003). Esto se debe a que cuando se habla de número absoluto de partículas, sin considerar su tamaño, todas contribuyen de igual manera a la suma final; sin embargo, se debe tener en cuenta que las partículas más grandes indicarían la ocurrencia de fuegos, o bien más severos, o bien más cercanos, aportando por tanto una información diferente a la de las partículas pequeñas. Para realizar esta transformación se ha seguido el método propuesto por Tinner y Hu (2003), que basa el cálculo de la concentración en la ecuación 1.

$$
\ln A=-7.418+9.36 \ln N
$$

siendo A la concentración en $\mathrm{mm}^{2} / \mathrm{g}$ y $\mathrm{N}$ la concentración en $\mathrm{n}^{\circ}$ partículas/g. La concentración en $n^{\circ}$ partículas/g se calcula siguiendo la ecuación 2 .

$$
\text { Cálculo de } \mathrm{n}^{0} \text { de partículas de microcarbón } / \mathrm{g}=\frac{\mathrm{n}^{0} \text { de partículas } \bullet \text { total de esporas de Lycopodium }}{\mathrm{n}^{0} \text { de esporas de Lycopodium contadas } \bullet \text { peso de la muestra }}
$$


Con ayuda del programa PAST (PAlaeontologicalSTatistics) (Hammer et al., 2001) se ha realizado una correlación no paramétrica, utilizando el índice de Spearman ( $\mathrm{r}$ de Spearman) entre las diferentes especies y el área de carbón en cada muestra para poder conocer así posibles relaciones entre fuego y vegetación. La utilización de este índice se debe a que la respuesta de los taxones a variables ambientales puede no ser unimodal en todos los casos. Algunos tipos polínicos se han agrupado para facilitar su análisis (Tabla 1).

Tabla 1. Agrupaciones de diferentes taxones representados en el diagrama polínico (Fig. 4).

\begin{tabular}{|c|c|c|c|}
\hline Mesófitos & Taxones adaptados a la aridez & Herbáceas heliófilas & Herbáceas ruderales \\
\hline Alnus & Ephedra distachya & Apiaceae & Fabaceae \\
\hline Betula & Ephedra fragilis & Artemisia & Papaver \\
\hline Carpinus & Tamarix & Asteroideae & Plantago \\
\hline Corylus & Centaurea & Boraginaceae & Rumex \\
\hline Fraxinus & Chenopodiaceae & Campanulaceae & Urticaceae \\
\hline Juglans & & Cardueae & \\
\hline Populus & & Caryophyllaceae & \\
\hline Salix & & Cichorioideae & \\
\hline \multirow[t]{13}{*}{ Ulmus } & & Cistaceae & \\
\hline & & Convolvulaceae & \\
\hline & & Genista & \\
\hline & & Gentiana & \\
\hline & & Helianthemum & \\
\hline & & Lamiaceae & \\
\hline & & Lotus type & \\
\hline & & Ribes & \\
\hline & & Rosaceae & \\
\hline & & Saxifragaceae & \\
\hline & & Scrophulariaceae & \\
\hline & & Sedum & \\
\hline & & Trifoliumtype & \\
\hline
\end{tabular}

\section{Resultados}

\subsection{Cronología y registro polínico}

El sondeo de la Basa de la Mora registra los últimos 10000 años de cambio ambiental (Pérez-Sanz, en prensa). Se han analizado un total de 142 muestras para la reconstrucción de la vegetación (Pérez-Sanz, en prensa), contándose en cada muestra un mínimo de 300 granos de polen y esporas, que contengan al menos 20 taxones diferen- 


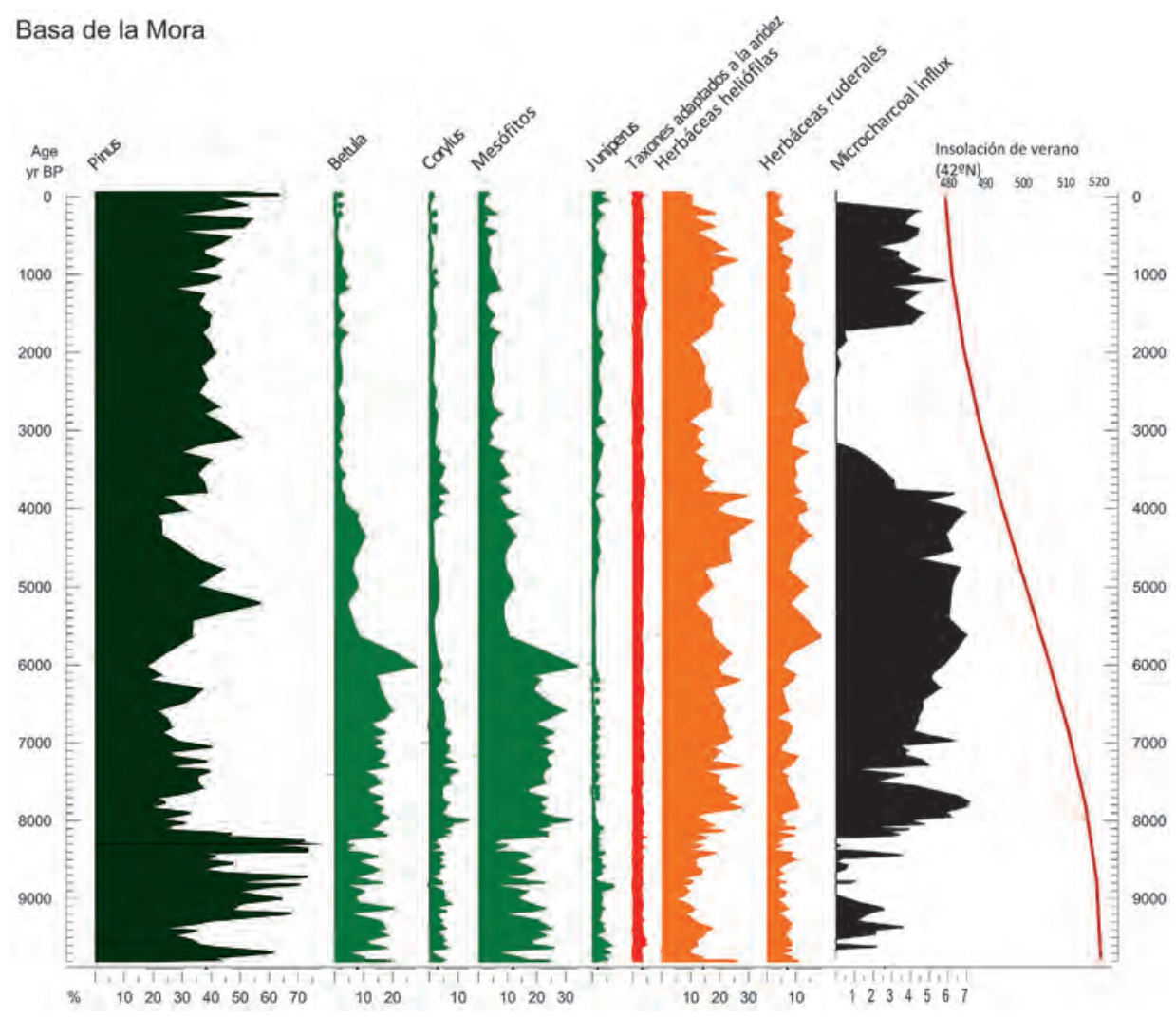

Figura 4. Representación de los géneros más importantes para la discusión (Pérez Sanz, en prensa), área de microcarbón $\left(\mathrm{mm}^{2} / \mathrm{g}\right)$ e insolación de verano.

tes. En la Fig. 4 se representan los resultados del espectro polínico de una selección de taxones que nos interesan para la discusión, por su posible conexión con el fuego. Los grupos y taxones representados son aquellos que poseen un índice de correlación significativo con el área de carbón (Tabla 1).

\subsubsection{Microcarbones}

Se han contado 142 muestras para el estudio de microcarbones, pudiéndose observar una alta variabilidad entre las diferentes muestras (Fig. 5): número máximo de partículas $=3098$; número mínimo de partículas $=0$; número medio de partículas $=307.1$; $\mathrm{SD}=453.8$.

Los resultados obtenidos permiten establecer distintas zonas, según la variabilidad y producción de partículas de microcarbones. En la tabla 2 se detallan los resultados obtenidos en la secuencia de microcarbón fósil. 
Tabla 2. Zonas de cambio en el área de carbón en la secuencia de la Basa de la Mora.

\begin{tabular}{|c|c|c|c|}
\hline Zona & $\begin{array}{l}\text { Profundidad } \\
(\mathrm{cm})\end{array}$ & $\begin{array}{l}\text { Edad estimada } \\
\text { (años BP) }\end{array}$ & Descripción \\
\hline 1 & $1167.5-815$ & $9800-8100$ & $\begin{array}{l}\text { Este es un periodo de gran variabilidad en la activi- } \\
\text { dad de fuego regional. A pesar de esta gran variabi- } \\
\text { lidad la concentración de partículas de microcarbón } \\
\text { nunca alcanza niveles muy altos (máx. no. part. } \\
\left(\mathrm{mm}^{2} / \mathrm{g}\right)=3.6 \text { y mín. }=0 ; \mathrm{SD}=1.00 \text { ). }\end{array}$ \\
\hline 2 & $815-616.5$ & $8100-7000$ & $\begin{array}{l}\text { La variabilidad sigue siendo muy alta pero la tasa de } \\
\text { producción de partículas de microcarbón se incre- } \\
\text { menta (máx. }=7.07 \text { y mín. }=0.75 ; \mathrm{SD}=1.55 \text { ) }\end{array}$ \\
\hline 3 & $616.5-392$ & $7000-4000$ & $\begin{array}{l}\text { Incremento en la actividad de fuegos a nivel regio- } \\
\text { nal. La concentración de partículas de microcarbón } \\
\text { se mantiene prácticamente constante a lo largo de todo } \\
\text { el periodo manteniendo valores muy altos de actividad } \\
\text { del fuego (máx } \mathrm{n}^{\circ} \text { part. }\left(\mathrm{mm}^{2} / \mathrm{g}\right)=6.96 \text { y mín }=4.14 \text {; } \\
\mathrm{SD}=0.92) \text {. }\end{array}$ \\
\hline 4 & $392-313.5$ & $4000-3200$ & $\begin{array}{l}\text { Declive en la actividad del fuego regional llegando } \\
\text { hasta una concentración nula alrededor de } 3.200 \text { años } \\
\text { BP. Durante este periodo la tasa máxima es de } 6.33 \\
\text { y la mínima de } 1.08 \text { partículas } \mathrm{mm}^{2} / \mathrm{g} \text {. Los valores } \\
\text { máximos se encuentran al inicio de la zona y los mí- } \\
\text { nimos en el final. } \mathrm{SD}=1.69 \text {. }\end{array}$ \\
\hline 5 & $313.5-200$ & $3200-1700$ & $\begin{array}{l}\text { La falta de microcarbones en esta sección nos hace } \\
\text { considerar que no se han producido incendios en esta } \\
\text { época. Nunca se alcanza en este periodo un valor de } \\
\text { área de microcarbones mayor a } 0.53 . \mathrm{SD}=0.23 \text {. }\end{array}$ \\
\hline 6 & $200-0$ & 1700-presente & $\begin{array}{l}\text { Aumento dramático de la concentración de microcar- } \\
\text { bones, siendo de nuevo alta tanto la variabilidad como } \\
\text { la tasa de producción de partículas (máx } \mathrm{n}^{\circ} \text { part. } \\
\left.\left(\mathrm{mm}^{2} / \mathrm{g}\right)=5.88 \text { y mín }=2.19 ; \mathrm{SD}=0.79\right)\end{array}$ \\
\hline
\end{tabular}

\subsubsection{Correlaciones fuego-vegetación}

En la tabla 3 se pueden observar los resultados significativos $(\mathrm{p}<0.05)$ de la correlación realizada entre el área de microcarbón y los diferentes taxones o agrupamientos vegetales (Tabla 1). Los únicos taxones que poseen una correlación negativa con el fuego son Pinus y Juniperus (-0.63 y -0.545, respectivamente). Por otro lado, el resto de taxones o grupos estudiados que mantienen una correlación significativa, poseen una correlación positiva con la ocurrencia de fuegos. Cabe destacar que los valores más altos son los obtenidos en los casos de las herbáceas heliófilas $(0.62)$, las comunidades mesófitas (0.42), y Betula (0.40). 
Tabla 3. Índices de correlación entre el área de microcarbón y las diferentes especies vegetales del registro de la Basa de la Mora.

\begin{tabular}{|l|c|}
\hline Taxones/Grupos & Índice de correlación Spearman $\mathbf{r}_{\mathbf{s}}$ \\
\hline Arbustos aridez & 0.26 \\
\hline Herbáceas heliófilas & 0.62 \\
\hline Taxones adaptados a la aridez & 0.23 \\
\hline Mesófitos & 0.42 \\
\hline Pinus & -0.63 \\
\hline Juniperus & -0.54 \\
\hline Betula & 0.40 \\
\hline Corylus & 0.26 \\
\hline
\end{tabular}

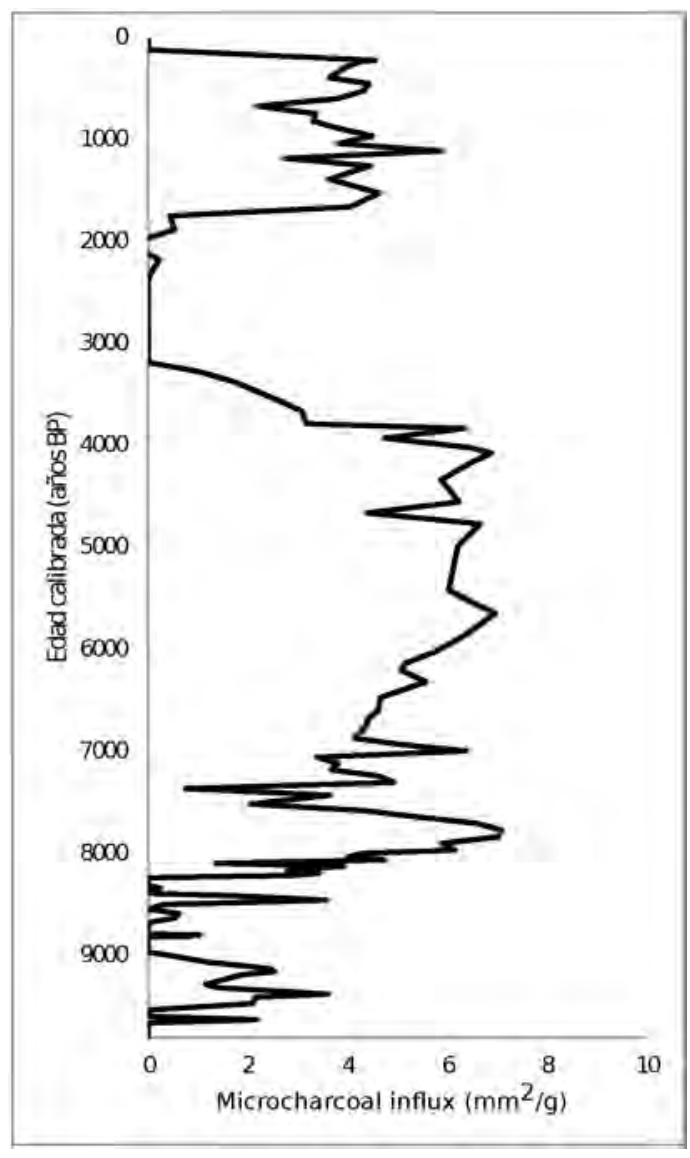

Figura 5. Representación del área de microcarbones $\left(\mathrm{mm}^{2} / \mathrm{g}\right)$ a partir de los conteos realizados. 


\section{Discusión}

\subsection{Influencia del clima en la ocurrencia de fuegos}

El comienzo del Holoceno se caracteriza, regional y continentalmente (Roberts, 1998; Labeyrie et al., 2003), por un progresivo calentamiento del clima que, en consecuencia, habría favorecido el aumento de la actividad del fuego. Los cambios precesionales, que afectan a la insolación estacional, explicarían que la mayor insolación durante el verano existente al comienzo del Holoceno diera lugar, entre otros factores, a cambios en la vegetación y en la ocurrencia de incendios (Fig. 4).

Entre ca. 9.8 cal Ka BP y 8.1 cal Ka BP la actividad de fuego es variable y no alcanza en ningún momento valores demasiado altos. Es a partir de $8.1 \mathrm{cal} \mathrm{Ka} \mathrm{BP} \mathrm{cuan-}$ do, en el registro de la Basa de Mora (BSM), se observa un aumento en la ocurrencia de incendios, con mayor variabilidad e intensidad hasta 7 cal Ka BP y una mayor constancia hasta el 4 cal Ka BP. Este incremento en la frecuencia e intensidad del fuego, a nivel milenario, podría estar relacionado con las consecuencias que el Óptimo Climático Holoceno (registrado en BSM entre 8.1 y 5.7 cal Ka BP), tuvo en la cubierta vegetal. Este es un periodo de creciente temperatura y relativa humedad (Roberts, 1998; Davis et al., 2003), que habría permitido el rápido crecimiento de comunidades mesófitas, teniendo como consecuencia el aumento de la biomasa disponible para ser quemada. La idea de una mayor humedad ligada a una intensificación del fuego puede resultar un tanto contra-intuitiva. Sin embargo, un estudio reciente realizado por Pausas y Paula (2012), corrobora la idea de que el combustible disponible ante un posible incendios forestal puede ser un factor limitante importante en algunas zonas, ya que por muy áridas que sean las condiciones climáticas, difícilmente se producirá un gran incendio en un área desprovista de vegetación. El trabajo de Pausas y Paula (2012) confirma que, en zonas de bosque húmedo asociadas a una alta productividad vegetal, una pequeña reducción en las condiciones de humedad, un episodio de aridez o una estación inusualmente seca, favorecerían un aumento en la actividad del fuego. Este modelo de producción de incendios se ajusta a la dinámica observada en la Basa de la Mora -una zona de alta montaña, cercana al límite del bosque, donde la cobertura leñosa puede verse limitada-. Así, durante la transición del Holoceno Temprano al Holoceno Medio (ca. 8-5.7 cal Ka BP), un clima más húmedo y cálido que el encontrado en el periodo anterior, junto con un estiaje más intenso debido a una mayor insolación, habría dado lugar a la acumulación de biomasa disponible, ocasionando fuegos más violentos y extensos durante los periodos secos.

En este sentido, hay que destacar que el descenso en la actividad del fuego que se observa en el registro de la BSM a partir de 4 cal Ka BP, coincide con una clara disminución en la curva de insolación de verano (Fig. 4), que reduciría el estiaje y provocaría condiciones climáticas menos favorables a la ocurrencia de incendios. Además, se produjo un retroceso del bosque mesófito, que también pudo influir en la disminución de la actividad del fuego, al reducirse la biomasa disponible como combustible. 
A partir de 1.7 cal Ka BP, con valores mínimos de insolación de verano, se registra un nuevo incremento de la actividad del fuego en la región que, probablemente, ya no está controlada por el clima, sino por un aumento en las actividades humanas. A pesar de que en los alrededores de la Basa de la Mora el impacto antropogénico se considera relativamente leve hasta hace aproximadamente 700 años (Pérez-Sanz et al., 2011), no se puede descartar que hubiera una señal regional más amplia que respondiera a los incendios ocurridos desde $1.7 \mathrm{cal} \mathrm{ka} \mathrm{BP}$.

A lo largo de la secuencia de la Basa de la Mora, se observa cierto grado de interacción entre los espectros polínicos y de microcarbones. Para facilitar su discusión, se detallan a continuación las posibles relaciones que existen entre fuego y vegetación.

\subsection{Pinus}

Las especies del género Pinus que podemos encontrar actualmente en el entorno de la Basa de la Mora (Pinus nigra y Pinus sylvestris), no serían capaces de regenerarse correctamente tras la ocurrencia de fuegos intensos, ya que no son especies rebrotadoras y además sus frutos no son serótinos (Escudero et al., 1999; Eshel et al., 2000; Tapias et al., 2004; Paula y Pausas, 2009; Paula et al., 2009). El presente estudio, muestra también esta falta de estrategia post-incendio entre los tipos polínicos de Pinus registrados, dada la correlación negativa existente entre la abundancia de este taxón y el área de microcarbón (Tabla 3). Además, se puede observar en la Fig. 4 que en el momento en el que los incendios son más intensos, se produce un importante retroceso de Pinus (8.1-5 cal Ka BP), que probablemente conformaría los bosques más cercanos al lago.

\subsection{Juniperus}

Diferentes autores (Riera y Castell, 1997; Pausas, 1999; Pausas et al., 2008) coinciden en el hecho de que el género Juniperus no presenta ninguna estrategia de respuesta al fuego. Esto se corrobora en el presente estudio, debido al alto índice de correlación negativo existente entre Juniperus y el área de microcarbón (Tabla 3), que nos indica que las poblaciones de Juniperus se verían reducidas tras la ocurrencia de un fuego.

Además, podríamos considerar que la aparición de Juniperus en una alta abundancia al comienzo del Holoceno, se debería principalmente al clima variable que dominaba en esos momentos. Este género puede soportar temperaturas de hasta $-25^{\circ} \mathrm{C}$ en invierno y de $40^{\circ} \mathrm{C}$ en verano, así como la sequía, por lo que podemos considerarla una especie bien adaptada para este momento de transición del Holoceno temprano. Lo más probable es que se tratara de Juniperus thurifera L. (sabina albar) y/o, con mayor certeza, Juniperus communis L. (enebro), ya que es una especie que suele situarse entre 1000 y $1800 \mathrm{~m}$ de altitud, en zonas de importante continentalidad, donde el suelo es pobre, poco desarrollado, y las lluvias son escasas, impidiendo la expansión de otras especies más exigentes (Blanco-Castro et al., 1997). 


\subsection{Mesófitos}

En el análisis de correlaciones, se observa una relación positiva entre las comunidades de bosque mesófito y la actividad del fuego a nivel regional (Tabla 3). Las características climáticas del Óptimo Climático Holoceno habrían determinado el rápido crecimiento de estas comunidades, permitiendo la existencia de gran cantidad de biomasa combustible, que favorecería la ocurrencia y rápida propagación de los fuegos (Pausas, 2004; Tinner y Lotter, 2006). El bosque mesófito, además de estar favoreciendo la propagación de los fuegos, sería capaz de colonizar rápidamente los espacios vacíos creados por el propio incendio, ya que está compuesto por taxones anemócoros -dispersión de semillas por el viento-, con estrategias post-incendio bien descritas, como el rebrote de cepa, resultando por ello fuertemente competitivos frente a otros taxones, perjudicados por las perturbaciones (Paula y Pausas, 2009; Paula et al., 2009; Gil-Romera et al., 2010). El retroceso de las poblaciones de bosque mesófito que se registra en BSM a partir de 5.7 cal Ka BP, podría deberse al alcance de un umbral de resiliencia, a partir del cual estas poblaciones se habrían visto gravemente dañadas y no habrían sido capaces de seguir regenerándose como hasta este momento, puesto que la frecuencia, o la intensidad de los eventos de fuego, habrían afectado a su capacidad de recuperación.

Dentro de las especies mesófitas que encontramos en la Basa de la Mora, hay que destacar la gran representación de Betula y Corylus.

El abedul (Betula spp.) presenta, además de las citadas características dispersivas y de estrategia post-incendio, un rápido crecimiento, lo que lo convierte en un taxón pionero (Blanco-Castro et al., 1997). Es sabido que los abedules son especies altamente competitivas en situaciones pioneras, porque se expanden rápidamente cuando las condiciones ambientales son favorables, instalándose en lugares donde los robledales, hayedos, pinares o abetales, han sido destruidos por causas antropogénicas o naturales (Blanco-Castro et al., 1997). La ecología de este taxón apoya por tanto el índice de correlación positivo entre Betula y el área de microcarbones, de modo que una mayor actividad del fuego, habría favorecido la expansión del abedul, en lugares que estaban anteriormente ocupados por otras especies vegetales. Esta relación ha sido ya comprobada por otros autores como Tinner et al. (1999) y Morales-Molino et al. (2011), que han observado el incremento de Betula tras la actividad del fuego en los Alpes suizos y en el NW de la Península Ibérica, respectivamente.

La expansión del avellano (Corylus avellana), también se ve favorecida por un aumento en la actividad del fuego, tanto en la Basa de la Mora presentando una correlación positiva con la actividad del fuego, como en numerosas zonas de montañas Mediterráneas (Tinner et al., 1999, 2005; Tinner y Lotter, 2001; Finsinger et al., 2006; Vannière et al., 2008). El incremento de Corylus asociado a altas actividades de fuego, se debe a que esta especie, también representa estrategias post-incendio, pudiendo rebrotar tras un fuego, y considerándosela también una especie pionera (Finsinger et al., 2006). En la secuencia estudiada, se observa una disminución de Corylus a partir de 5.7 ka BP, momento en el que consideramos que se habría llegado al mencionado umbral de resiliencia, a partir del cual estas comunidades no habrían sido capaces de restablecerse; bien porque la dinámica 
del fuego supera su capacidad de regeneración, bien porque las condiciones climáticas no resultaban tan favorables. El hecho de que el avellano experimente una pequeña expansión a partir de 4000 BP, cuando disminuye la actividad del fuego, apoya la idea de una respuesta umbral del avellano y otros mesófitos entorno al 5.7 ka BP. Por el contrario, el aumento de Corylus ca. 4 ka BP podría deberse a un clima más cálido, que favoreciese la expansión del avellano frente a la del abedul, y/o a la competitividad de esta especie frente a otras existentes ante la todavía importante actividad del fuego.

\subsection{Herbáceas y taxones adaptados a la aridez}

La importancia del fuego en los ecosistemas donde predominan las especies herbáceas, ha sido extensamente descrita por Bond et al. (2005), lo que se ve apoyado por el presente estudio, donde la dinámica de las herbáceas heliófilas es muy similar a la que sigue la actividad del fuego. A partir de $8.1 \mathrm{cal} \mathrm{Ka} \mathrm{BP,} \mathrm{se} \mathrm{produce} \mathrm{una} \mathrm{expansión} \mathrm{en} \mathrm{el}$ conjunto de los taxones que comprenden este grupo, paralelo al periodo de mayor ocurrencia de incendios. Así, las herbáceas heliófilas, parecen responder positivamente al efecto del fuego entre 8.1 y 4 cal Ka BP, y de nuevo a partir de 1.7 cal Ka BP, hasta la actualidad (Tabla 3). Esta relación puede estar vinculada a que el fuego crea espacios abiertos en los que penetra mucha luz, y la colonización de este tipo de especies se ve, por tanto, facilitada.

Aunque no parece existir una respuesta al fuego tan clara en las herbáceas ligadas a la perturbación, sí que existe una relación positiva significativa entre ambas variables (Tabla 3). Esto nos permite asegurar que se da una importante relación entre la actividad del fuego y todas las plantas herbáceas, ya que, o bien éstas serían capaces de colonizar rápidamente los espacios abiertos por el fuego (Eshel et al., 2000), o bien permiten la propagación del fuego si no hay otro combustible disponible.

Por otro lado, la curva de los taxones adaptados a la aridez se mantiene prácticamente constante a lo largo de toda la secuencia, por lo que resulta difícil inferir relaciones de este grupo con la actividad del fuego. El resultado positivo de la correlación entre estas dos variables (Tabla 3), se podría explicar por una covariación debido al clima árido que modula ambos factores: fuegos y taxones adaptados a la aridez. Además, al igual que los grupos de herbáceas anteriores, estos taxones pueden ser considerados pioneros porque responden rápidamente a las perturbaciones, ocupando los nuevos espacios creados (Eshel et al., 2000).

\section{Conclusiones}

En este trabajo se presenta la dinámica de la actividad del fuego durante los últimos diez mil años en el Pirineo central, su relación con algunas de las especies vegetales que han estado presentes durante este tiempo, y su relación con el clima. De este estudio se extraen las siguientes conclusiones: 
1. La actividad del fuego en la Basa de la Mora parece estar estrechamente ligada a la dinámica precesional, desde el Holoceno Temprano hasta el 1700 BP, cuando el papel de la actividad humana podría ser más relevante. Los periodos de mayor insolación dan lugar a estiajes más intensos y, consecuentemente, a una mayor actividad del fuego, que a su vez, es modulada por la alta biomasa disponible entre 8.1 y 4 cal Ka BP. A partir de 4 cal Ka BP, el descenso de la insolación de verano produciría una menor incidencia de fuegos, llegando a ser prácticamente nula entre 3.2 y 1.7 cal Ka BP, lo que coincide con la contracción del bosque mesófito, que podría haber alcanzado una respuesta umbral al efecto del fuego.

2. Se observan relaciones negativas entre la ocurrencia de fuego, Juniperus y Pinus. La influencia negativa del fuego sobre estos taxones se debe, principalmente, a la ausencia de una estrategia de respuesta al fuego.

3. Betula, Corylus, herbáceas y taxones adaptados a la aridez presentan correlaciones positivas con el fuego. Estos taxones sí tienen estrategias de adaptación a la perturbación, como por ejemplo, la capacidad rebrotadora de Corylus, o la efectividad de colonización de espacios abiertos de Betula, y de determinadas herbáceas.

Los resultados obtenidos en este trabajo, vienen a confirmar el relevante papel de la biomasa en la ocurrencia de incendios, poniendo de manifiesto la necesidad de tener en cuenta las relaciones entre clima, vegetación y fuegos en escalas temporales largas, para poder generar un conocimiento integral que sirva para la gestión de la naturaleza en el actual escenario de Cambio Global.

\section{Agradecimientos}

Esta investigación ha sido financiada con los proyectos ARAFIRE Convenio DGAla Caixa ref. 2012 GA LC 064, DINAMO ref. CGL2009-07992, GRACCIE-CONSOLIDER ref. CSD2007-00067 y HORDA-OAPN ref: 83/2009.

\section{Referencias bibliográficas}

AEMET-IM, 2011. Atlas Climático Ibérico. Agencia Estatal de Meteorología Ministerio de Medio Ambiente y Medio Rural y Marino, Madrid.

Bal, M.C., Pelachs, A., Pérez-Obiol, R., Julià, R., Cunill, R. 2011. Fire history and human activities during the last $3300 \mathrm{cal}$ yr BP in Spain's Central Pyrenees: The case of the Estany de Burg. Palaeogeography, Palaeoclimatology, Palaeoecology 300, 179-190.

Belmonte-Ribas, A. 1999. Aportaciones a la geomorfología del Macizo de Cotiella (Pirineo Oscense). Lucas Mallada: Revista de Ciencias 11, 25-40.

Belmonte-Ribas, A. 2007. La cueva 5 de Agosto (Macizo de Cotiella, Pirineo de Huesca): Registros kársticos y consideraciones paleoambientales. Instituto de Estudios Altoaragoneses, Huesca, 65 pp. 
Bennett, K.D. 2009. Documentation for psimpoll 4.27 and pscomb 1.03: C programs for plotting pollen diagrams and analysing pollen data. Disponible en: http://chrono.qub.ac.uk/psimpoll/ psimpoll_manual/4.27/psimpoll.htm.

Blanco-Castro, E., Casado-González, M., Costa-Tenorio, M., Escribano-Bombín, R., García-Antón, M., Génova-Fuster, M., Gómez-Manzaneque, A., Gómez-Manzaneque, F., Moreno-Sáiz, J., Mora-Juaristi, C. 1997. Los bosques ibéricos. Una interpretación geobotánica. Planeta, Barcelona, $572 \mathrm{pp}$.

Bond, W.J., Keeley, J.E. 2005. Fire as a global "herbivore": the ecology and evolution of flammable ecosystems. Trends in Ecology \& Evolution 20, 387-394.

Bond, W.J., Woodward, F.I., Midgley, G.F. 2005. The global distribution of ecosystems in a world without fire. New Phytologist 165, 525-538.

Bowman, D.M.J., Balch, J.K., Artaxo, P., Bond, W.J., Carlson, J.M., Cochrane, M.A., D’ Antonio, C.M., Defries, R.S., Doyle, J.C., Harrison, S.P., Johnson, F.H., Keeley, J.E., Krawchuk, M.A., Kull, C.A., Marston, J.B., Moritz, M.A., Prentice, I., Roos, C.I., Scott, A.C., Swetnam, T.W., Van der Werf, G.R. 2009. Fire in the Earth system. Science 324, 481-484.

Carcaillet, C., Bouvier, M., Fréchette, B., Larouche, A.C., Richard, P.J.H. 2001. Comparison of pollen-slide and sieving methods in lacustrine charcoal analyses for local and regional fire history. The Holocene 11, 467-476.

Clarck, J.S. 1988. Particle motion and the theory of charcoal analysis: source area, transport, deposition, and sampling. Quaternary Research 30, 67-80.

Conedera, M., Tinner, W., Neff, C., Meurer, M., Dickens, A.F., Krebs, P. 2009. Reconstructing past fire regimes: methods, applications, and relevance to fire management and conservation. Quaternary Science Reviews 28, 555-576.

Davis, B.A.S., Brewer, S., Stevenson, A.C., Guiot, J. 2003. The temperature of Europe during the Holocene reconstructed from pollen data. Quaternary Science Reviews 22, 1701-1716.

Driscoll, D.A., Lindenmayer, D.B., Bennett, A.F., Bode, M., Bradstock, R.A., Cary, G.J., Clarke, M.F., Dexter, N., Fensham, R., Friend, G., Gill, M., James, S., Kay, G., Keith, D.A., MacGregor, C., Rusell-Smith, J., Saly, D., Watson, J.E.M., Williams, R.J. 2010. Fire management for biodiversity conservation: Key research questions and our capacity to answer them. Biological Conservation 143, 1928-1939.

Dupré, M. 1979. Breve manual de análisis polínico. Instituto Juan Sebastián Elcano, CSIC, Valencia. Escudero, A., Sanz, M.V., Pita, J.M., Pérez-García, F. 1999. Probability of germination after heat treatment of native Spanish pines. Annals of Forest Science 56, 511-520.

Eshel, A., Henig-Sever, N., Ne'eman, G. 2000. Spatial variation of seedling distribution in an east Mediterranean pine woodland at the beginning of post-fire succession. Plant Ecology 148, 175-182.

Finsinger, W., Tinner, W., Van der Knaap, W.O., Ammann, B. 2006. The expansion of hazel (Corylusavellana L.) in the southern Alps: a key for understanding its early Holocene history in Europe? Quaternary Science Reviews 25, 612-631.

Fisher, J.L., Loneragan, W.A., Dixon, K., Delaney, J., Veneklaas, E.J. 2009. Altered vegetation structure and composition linked to fire frequency and plant invasion in a biodiverse woodland. Biological Conservation 142, 2270-2281.

Gil-Romera, G., Carrión, J.S., Pausas, J.G., Sevilla-Callejo, M., Lamb, H.F., Fernández, S., Burjachs, F. 2010. Holocene fire activity and vegetation response in South-Eastern Iberia. Quaternary Science Reviews 29, 1082-1092.

Gottfried, M., Pauli, H., Futschik, A., Ahalkatsi, M., Barankok, P., Alonso, J.L.B., Coldea, G., Dick, J., Erschbamer, B., Calzado, M.R.F., Kazakis, G., Krajci, J., Larsson, P., Mallaun, M., Michelsen, O., Moiseev, D., Moiseev, P., Molau, U., Merzouki, A., Nagy, L., Nakhutsrishvili, G., Pedersen, B., Pelino, G., Puscas, M., Rossi, G., Stanisci, A., Theurillat, J.-P., Tomaselli, M., 
Villar, L., Vittoz, P., Vogiatzakis, I., Grabherr, G. 2012. Continent-wide response of mountain vegetation to climate change. Nature Climate Change 2, 111-115.

Hammer, Ø., Harper, D.A.T., Ryan, P.D. 2001. PAST-Palaeontological statistics software package for education and data analysis.ver. 2.17b. Paleontologia Electronica 4 (1), 31.

IPCC 2007. Climate change 2007: The physical Science Basis. Cambridge University Press, Cambridge, $996 \mathrm{pp}$.

Keeley, J.E., Rundel, P.W. 2005. Fire and the Miocene expansion of C4 grasslands. Ecology Letters 8, 683-690.

Labeyrie, L., Cole, J., Alverson, K., Stocker, T. 2003. The History of Climate Dynamics in the Late Quaternary. En Paleoclimate, Global Change and the Future, K.D. Alverson, R.S. Bradley, T.F. Pedersen (eds.), Springer, New York, pp. 33-71.

Mann, M.E., Bradley, R.S., Hughes, M.K. 1999. Northern hemisphere temperatures during the past millennium: inferences, uncertainties, and limitations. Geophysical Research Letters 26 , 759-762.

Montserrat-Martí, G. 1989. Paisaje vegetal y síntesis de la vegetación del macizo de Cotiella y la Sierra de Chía (Prepirineo Central de Huesca). Lucas Mallada: Revista de Ciencias 1, 119-132.

Moore, P.D., Webb, J.A. 1978. An illustrated guide to pollen analysis. Hodder \& Stoughton, London.

Morales-Molino, C., García-Antón, M., Morla, C. 2011. Late Holocene vegetation dynamics on an Atlantic-Mediterranean mountain in NW Iberia. Palaeogeography, Palaeoclimatology, Palaeoecology 302, 323-337.

Niklasson, M., Granstrom, A. 2000. Numbers and Sizes of Fires: Long-Term Spatially Explicit Fire History in a Swedish Boreal Landscape. Ecology 81, 1484-1499.

Patterson, W.A., Edwards, K.J., Maguire, D.J. 1987. Microscopic charcoal as a fossil indicator of fire. Quaternary Science Reviews 6, 3-23.

Paula, S., Pausas, J.G. 2009. BROT: a plant trait database for Mediterranean Basin species. Disponible en: http://www.uv.es/jgpausas/brot.htm.

Paula, S., Arianotsou, M., Kazanis, D., Tavsanoglu, Ç., Lloret, F., Buhk, C., Ojeda, F., Luna, B., Moreno, J.M., Rodrigo, A. 2009. Fire-related traits for plant species of the Mediterranean Basin. Ecology 90, 1420-1420.

Pausas, J.G. 1999. Mediterranean vegetation dynamics: modelling problems and functional types. Plant Ecology 140, 27-39.

Pausas, J.G. 2004. Changes in fire and climate in the eastern Iberian Peninsula (Mediterranean basin). Climatic Change 63, 337-350.

Pausas, J.G., Keeley, J.E. 2009. A burning story: the role of fire in the history of life. BioScience 59, 593-601.

Pausas, J.G., Paula, S. 2012. Fuel shapes the fire-climate relationship: evidence from Mediterranean ecosystems. Global Ecology and Biogeography 21, 1074-1082.

Pausas, J.G., Llovet, J., Rodrigo, A., Vallejo, R. 2008. Are wildfires a disaster in the Mediterranean basin?: A review. International Journal of Wildland Fire 17, 713-723.

Pérez-Sanz, A. En prensa. Holocene climate variability, vegetation dynamics and fire regimen in the Central Pyrenees: the Basa de la Mora sequence (NE Spain). Quaternary Science Reviews.

Pérez-Sanz, A., González-Sampériz, P., Valero-Garcés, B.L., Moreno, A., Morellón, M., Sancho, C., Belmonte, A., Gil-Romera, G., Sevilla, M., Navas-Izquierdo, A. 2011. Clima y actividades humanas en la dinámica de la vegetación durante los últimos 2000 años en el Pirineo Central: el registro palinológico de la Basa de la Mora (Macizo de Cotiella). Zubía 23, 17-38.

Piñol, J., Terradas, J., Lloret, F. 1998. Climate warming, wildfire hazard, and wildfire occurrence in coastal eastern Spain. Climatic Change 38, 345-357.

Pyne, S.J., Andrews, P.L., Laven, R.D. 1984. Introduction to wildland fire. John Wiley and Sons, New York. 
Rhodes, A.N. 1998. A method for the preparation and quantification of microscopic charcoal from terrestrial and lacustrine sediment cores. The Holocene 8, 113-117.

Riera, J., Castell, C. 1997. Efectes dels incendis forestals recurrents sobre la distribució de dues espècies del Parc Natural del Garraf: el pi blanc (Pinus halepensis) i la savina (Juniperus phoenicea). Butll. Inst. Cat. Hist. Nat. 65, 105-116.

Rius, D., Vannière, B., Galop, D. 2009. Fire frequency and landscape management in the northwestern Pyrenean piedmont, France, since the early Neolithic (8000 cal. BP). The Holocene 19, 847-859.

Rius, D., Vannière, B., Galop, D., Richard, H. 2011. Holocene fire regime changes from multiplesite sedimentary charcoal analyses in the Lourdes basin (Pyrenees, France). Quaternary Science Reviews 30, 1696-1709.

Rius, D., Vannière, B., Galop, D. 2012. Holocene history of fire, vegetation and land use from the central Pyrenees (France). Quaternary Research 77, 54-64.

Roberts, N. 1998. The Holocene: an environmental history. Wiley-Blackwell, Malden.

Sadori, L., Giardini, M. 2007. Charcoal analysis, a method to study vegetation and climate of the Holocene: The case of Lago di Pergusa (Sicily, Italy). Geobios 40, 173-180.

Tapias, R., Climent, J., Pardos, J.A., Gil, L. 2004. Life histories of Mediterranean pines. Plant Ecology 171, 53-68.

Thonicke, K., Venevsky, S., Sitch, S., Cramer, W. 2001. The role of fire disturbance for global vegetation dynamics: coupling fire into a Dynamic Global Vegetation Model. Global Ecology and Biogeography 10, 661-677.

Tinner, W., Lotter, A.F. 2001. Central European vegetation response to abrupt climate change at 8.2 ka. Geology 29, 551-554.

Tinner, W., Hu, F.S. 2003. Size parameters, size-class distribution and area-number relationship of microscopic charcoal: relevance for fire reconstruction. The Holocene 13, 499-505.

Tinner, W., Lotter, A.F. 2006. Holocene expansions of Fagus sylvatica and Abies alba in Central Europe: where are we after eight decades of debate? Quaternary Science Reviews 25, 526-549.

Tinner, W., Hubschmid, P., Wehrli, M., Ammann, B., Conedera, M. 1999. Long-term forest fire ecology and dynamics in southern Switzerland. Journal of Ecology 87, 273-289.

Tinner, W., Conedera, M., Ammann, B., Lotter, A.F. 2005. Fire ecology north and south of the Alps since the last ice age. The Holocene 15, 1214-1226.

Vannière, B., Colombaroli, D., Chapron, E., Leroux, A., Tinner, W., Magny, M. 2008. Climate versus human-driven fire regimes in Mediterranean landscapes: the Holocene record of Lagodell'Accesa (Tuscany, Italy). Quaternary Science Reviews 27, 1181-1196.

Whitlock, C., Higuera, P.E., McWethy, D.B., Briles, C.E. 2010. Paleoecological Perspectives on Fire Ecology: Revisiting the Fire-Regime. TOECOLJ 3, 6-23.

Whitlock, C., Larsen, C. 2001. Charcoal as a Fire Proxy. En Tracking Environmental Change Using Lake Sediments, J. P. Smol, H. J. Birks, W. M. Last, R. S. Bradley, K. Alverson (eds.), Kluwer Academic Publishers, Dordrecht, pp. 75-97. 